\title{
Der Sportarztkoffer
}

\author{
Casper Grim, Martin Engelhardt
}

\section{Zusammenfassung}

Was gehört eigentlich in den Arztkoffer? Der Wunsch, auf dem Spielfeld auf alle Eventualitäten vorbereitet zu sein und eine entsprechende Ausrüstung vorhalten zu können, entspricht häufig nicht der Realität. Dennoch gibt es viele hilfreiche Tools, die der Sportarztkoffer beinhalten kann. Es gibt nicht den einen Universalkoffer; vielmehr kann man von einer individuellen und variablen Zusammenstellung mit einigen Grundausstattungsmerkmalen sprechen. In der Sportbetreuung ist auch die Kenntnis der AntiDoping-Richtlinien obligat. Im diesem Beitrag sollen dem Leser einige Grundüberlegungen vermittelt und konkrete Tipps für die Zusammenstellung eines Sportarztkoffers gegeben werden.

\section{Team Physicians Bag}

What should the team physician pack in his bag? In the real world, it is not always possible to be prepared for all circumstances and to have all equipment that you might possibly need. Nevertheless, there are many useful tools that a sports medicine bag can contain. There are no general rules, but rather an individual and variable content with some basic features. In sports medicine, knowledge of antidoping policy is obligatory. The present article will present some general principles and specific advice for packing a sports medicine bag.

\section{Einleitung}

Jeder Sportinteressierte hat es schon häufiger gesehen: Nach einem Foulspiel liegt der Spieler am Boden und kann nicht mehr aufstehen. Der Mannschaftsarzt und der Physiotherapeut rennen auf den Platz, um den verletzten Sportler zu behandeln. Dabei ist meist eine Tasche voller Utensilien. Der Wunsch, auf alle Eventualitäten vorbereitet $\mathrm{zu}$ sein und eine entsprechende Ausrüstung vorzuhalten entspricht hierbei häufig nicht der Realität. Dennoch gibt es viele hilfreiche Tools, die der Sportarztkoffer beinhalten kann.

Für viele im Bereich der Sportmedizin Tätige stellt sich bei der medizinischen Betreuung und Versorgung die Frage: Was gehört eigentlich in den Arztkoffer?

OP-JOURNAL 2016; 32: 6-9

(c) Georg Thieme Verlag KG Stuttgart · New York DOI http://dx.doi.org/10.1055/s-0035-1568628
Zunächst sollte bereits an dieser Stelle klargestellt werden, dass der Sportarztkoffer anders zusammengestellt ist als die Betreuungskoffer für bspw. den Sportphysiotherapeuten oder den Trainer/Betreuer. Trotzdem gibt es u.a. für die „Erste-Hilfe“-Anforderungen hier auch viele Gemeinsamkeiten. Der wesentliche Unterschied beim Gebrauch eines Sportarztkoffers ist, dass die Ausgabe und Verordnung von Medikamenten ausschließlich dem Arzt obliegt.

Verschreibungspflichtige Medikamente können und dürfen nur auf ärztliche Anordnung gegeben werden. Die Grundlagen hierfür finden sich im deutschen Arzneimittelgesetz und der Arzneimittelverschreibungsverordnung. Diese regeln den sachgemäßen Umgang mit verschreibungspflichtigen Arzneimitteln im Interesse einer ordnungsgemäßen und sicheren Arzneimittelanwendung. Dem gegenüber steht, dass ein Großteil der benötigten Utensilien nicht medikamentöser Art ist. Insbesondere sind hier unterschiedliche Verbandsmaterialien, Tapes, Salben und Eis zu nennen. Untersuchungen oder systematische Reviews über den notwendigen Inhalt des Sportarztkoffers gibt es verständlicherweise nicht. Im Wesentlichen handelt es sich somit um die Erfahrung des Einzelnen mit den verschiedenen Inhalten. Im Folgenden sollen dem Leser einige, hoffentlich hilfreiche, Tipps und Ausführungen dargestellt werden.

Ein wichtiger Faktor für die Zusammenstellung des Sportarztkoffers ist das „Setting“. So macht es verständlicherweise einen Unterschied, ob der Arzt ein einzelnes Fußballspiel oder ein ganzes, ggf. mehrwöchiges Turnier betreut. Ebenfalls gilt es eine Differenzierung für die verschiedenen Sportarten zu machen. So sollte der Inhalt des Sportarztkoffers bei Kampfsportarten durchaus unterschiedlich zu einem Betreuungskoffer für eine Ausdauersportart sein. Nasenbluten, Platzwunden, Luxationen und das Risiko für Halswirbelsäulenverletzungen spielen im Ausdauersport eine untergeordnete Rolle, können bei einigen Kampfsportarten aber zum „Tagesgeschäft“ gehören. Zusätzlich muss bedacht werden, dass der Umfang und Inhalt der Kofferausstattung für die Betreuung einer Trainingsmaßnahme typischerweise umfangreicher ist als für den Wettkampf. So kann aus dem Sportarztkoffer auch schnell ein 2. Koffer oder gar eine kleine Sportarztapotheke werden (Abb. 1 und 2).

Es gibt nicht den einen Universalkoffer; vielmehr kann man von einer individuellen und variablen Zusammenstellung mit einigen Grundausstattungsmerkmalen sprechen.

Erfahrungen in der jeweiligen Sportart und die Kenntnis der sportartspezifischen Anforderungsprofile sind für den betreuenden Arzt unumgänglich und nicht nur für die Zusammenstellung der Materialauswahl zwingend notwendig. 


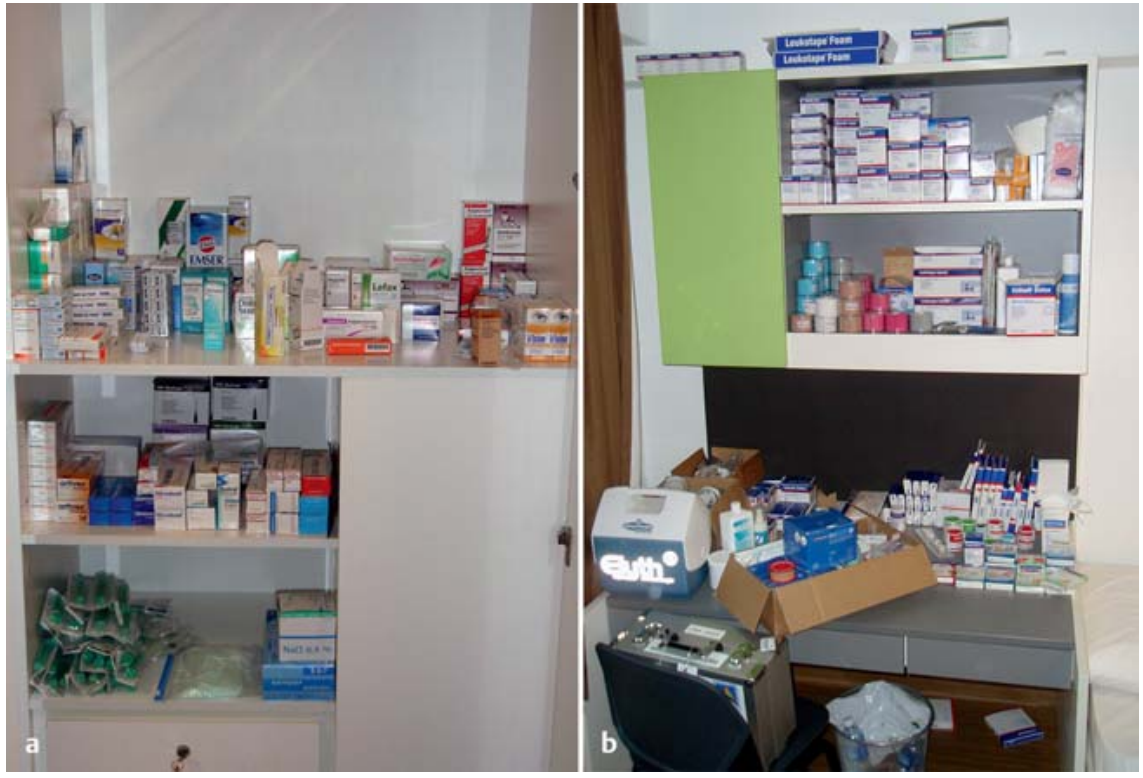

Abb. 1 a und b Beispiel einer Sportarztapotheke für die mehrtätige Mannschaftsversorgung bei einer Sportgroßveranstaltung. Medikamente und Verbands-Taping-Material.

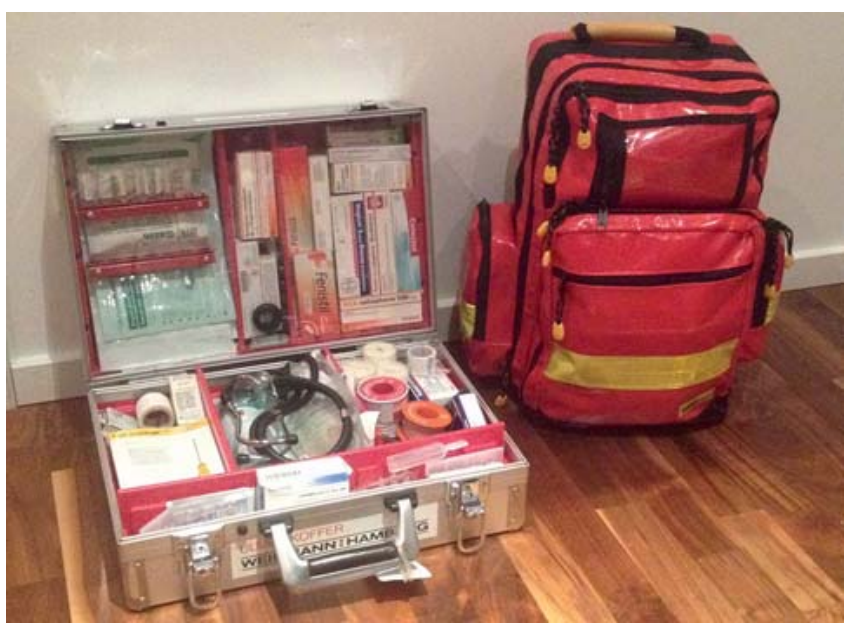

Abb. 2 Beispiel eines Sportarztkoffers mit Notfallrucksack.

Bei größeren Maßnahmen, bei denen mehrere Personen an der medizinischen Betreuung beteiligt sind, empfiehlt es sich, eine zentrale Koordination vorzunehmen, und es sollte eine Absprache bezüglich der Material- und Medikamentenauswahl mit den zuständigen Ärzten und Physiotherapeuten erfolgen. Dies gewährleistet eine optimale Ressourcennutzung und vermeidet Situationen, in denen einige Dinge doppelt und 3-fach, andere hingegen gar nicht vorhanden sind, da es in der jeweiligen Ausrüstung typischerweise Überschneidungen gibt.

Es empfiehlt sich, die „Apotheke“ selbst zusammenzustellen und mitzuführen, um bspw. nicht im Ausland Medikamente kaufen und besorgen zu müssen. Dies hat unterschiedliche Gründe. Ein relevanter Faktor ist sicherlich die unmittelbare Verfügbarkeit und der ohne Zeitverzöge- rung durchführbare Behandlungsbeginn bei der jeweiligen Betreuungsmaßnahme. Es sollte gewährleistet sein, dass der Arzt und ggf. auch der Athlet auf für ihn bekannte Medikamente zurückgreifen kann. Dies erhöht die Anwendungssicherheit und reduziert das Risiko einer Fehlmedikation oder Fehldosierung. Ebenfalls ist das Risiko einer Medikamentenverunreinigung, die in einigen Ländern nicht ausgeschlossen, vielleicht sogar wahrscheinlich ist, minimiert.

Die Ausrüstung ist häufig, u.a. aus logistischen Gründen, auf das Notwendige zu beschränken. Bei allen Medikamenten und dem Sterilgut (Spritzen, Nadeln etc.) ist das Verfallsdatum zu beachten und vor jedem Einsatz zu kontrollieren.

Packlisten für den Sportarztkoffer sind sinnvoll.

\section{Anti-Doping-Richtlinien}

Grundsätzlich sei darauf hingewiesen, dass in der Sportbetreuung selbstverständlich die Anti-Doping-Richtlinien gelten. Es ist darauf zu achten, dass keine Medikamente mitgeführt werden, die auf der Verbotsliste der Nationalen AntiDoping Agentur (NADA) und der World Anti-Doping Agency (WADA) stehen. Dies verhindert eine versehentliche Fehlmedikation mit schwerwiegenden Folgen. Ebenfalls ist auf die Art und Menge der Notfallmedikamente zu achten. So ist bspw. das Mitführen von mehreren Infusionsbestecken mit einer reinen Notfallversorgung wohl kaum zu erklären.

Der Sportarzt muss sich über die aktuellen Anti-Doping-Richtlinien informieren und ist inzwischen von den Sportverbänden angewiesen, die Teilnahme an entsprechenden Fortbildungsmaßnahmen nachzuweisen. Ein hilfreiches und praxisnahes Tool ist die NADA-App, die auf dem Smartphone oder dem Tablet nutzbar ist. Hier kann sehr einfach geprüft werden, welche Medikamente sicher zur Anwendung kommen können.

\section{Der Koffer}

Da es sich bei der akuten Behandlung mehr oder weniger um eine geplante „Erste Hilfe“ bei einer Sportverletzung handelt, sind ebenfalls Richtlinien und Vorschriften bez. der Minimalausstattung des Sportarztkoffers zu berücksichtigen. Diese sind in den DIN-Normen geregelt.

Der Anwendungsbereich kann sehr unterschiedlich sein und ist eben nicht eingeschränkt auf ein großes Fußballstadion. Für Schulen und Tageseinrichtungen für Kinder sollen, neben dem klassischen Verbandskasten, auch weitere „geeignete“ Verbandmittelbehältnisse genutzt werden, sofern sie die gleichen Eigenschaften aufweisen (Verschließbarkeit, Dichtigkeit, keine scharfen Ecken etc.). Der Sportarzt kann seine Utensilien also in einem geeigneten Sportarztkoffer mitnehmen und ist nicht auf einen Verbandskasten oder Verbandskoffer angewiesen.

Verbandskästen mit den Maßen 25,5× $16,6 \times 8 \mathrm{~cm}(\mathrm{~L} \times \mathrm{B} \times \mathrm{H})$ tragen die Bezeichnung „Verbandskasten DIN 13157-C“, andere Behälter die Bezeichnung „Inhalt DIN 13157“. Beides ist vom Gesetzgeber zur Anwendung freigegeben. 
Die DIN 13164 regelt die gesetzliche Anforderung für Fahrten zu Training und Spiel und entspricht lediglich dem KfzVerbandskasten, der ohnehin vorhanden sein sollte.

Die DIN 13157 beschreibt die notwendige Ausstattung nach Arbeitsschutzrichtlinien (ggf. auch Betriebssport). Die Verantwortlichen in Vereinen und im Betriebssport weisen mit diesem Kofferinhalt nach, dass alle Verbandmaterialanforderungen zum Schutz von Trainern, Betreuern, Spielern und Mitarbeitern zur Verfügung stehen. In den meisten Fällen sind hierfür der Veranstalter und der Sportstättenbetreiber und nicht der Arzt verantwortlich. Bei einigen Trainingslagern in durchaus abgelegenen Regionen, wie bspw. beim Wildwasserkanu, kann eine einfache Notfallversorgung allerdings schnell zur logistischen Herausforderung werden, und der Sportarzt ist gut beraten, eine eigene Grundausstattung mitzuführen (Abb. 2).

\section{Ausstattung}

Bezüglich der Erste-Hilfe-Ausstattung gibt es für Sportvereine keine spezifischen Vorschriften. Weder die Unfallverhütungsvorschrift „Grundsätze der Prävention“ (GUV-V A 1, insbesondere $\S \S$ 24-28) noch die Arbeitsstättenverordnung bestimmen, welches und wie viel Erste-Hilfe-Material im Einzelnen bereitzustellen ist. Gleiches gilt für den Sportarztkoffer. Hinzu kommt, dass der Arzt den Koffer meist ohnehin individuell zusammenstellt.

Von folgenden Richtwerten kann ausgegangen werden (Erste-Hilfe-Minimalausstattung):

- bis 50 Personen: kleiner Verbandskasten nach DIN 13157

- bis 300 Personen: großer Verbandskasten nach DIN 13169

- für je weitere 300 Personen: großer Verbandskasten nach DIN 13169

Der Inhalt des Verbandskastens nach DIN 13157 ist insgesamt 65-teilig und besteht aus folgenden Einzelkomponenten:

- Augenkompressen

- Fingerverbände

- Schere

- Dreiecktücher

- Heftpflaster

- Verbandpäckchen

- Einmalhandschuhe

- Verbandtücher

- Kältesofortkompresse

- Vliesstofftücher
- Kompressen

- Wundschnellverbände

- Fixierbinden

- Pflasterstrips

- Fingerkuppenverbände

- Rettungsdecke

- Folienbeutel

- Erste-Hilfe-Broschüre

- Inhaltsverzeichnis

Der Verbandskasten nach DIN 13169 unterscheidet sich vom Inhalt eines Verbandskastens nach DIN 13157 lediglich in der Menge der Produkte.

\section{Packliste für den Sportarztkoffer}

- Box mit Eiswasser und Schwämmen

- Kühlbox mit Eiswasser/Crush-Eis

- Kältespray, Chloräthyl-Spray

- Schwämme, ggf. Kompressionssystem

- entzündungshemmendes Gel, z.B. Voltaren Emulgel

- Heparinsalbe

- Wärmesalbe

- Mittel gegen Sonnenbrand, Allergie (z.B. Soventol ${ }^{\circledR}-/$ Fenistil $^{\circledR}-\mathrm{Gel}$ )

- Arnikasalbe: bei anhaltenden Muskelbeschwerden, Nachbehandlung von Zerrungen (z. B. Varicylum ${ }^{\circledR}$ S Salbe)

- Transpulmin ${ }^{\circledR}$ Balsam: Einreiben der Brust zum besseren Atmen, gerade im Winter

- Augen- und Nasensalbe: Läsionen der Augenbindehaut oder Nasenschleimhaut

- Fettcreme, Massageöl

- Desinfektionsmittel (zugelassen für Injektion/Wundversorgung, Schleimhaut, Händedesinfektion)

- Pflaster, Blasenpflaster, Pflastersprühverband

- Kompressen steril, Tupfer, Wattestäbchen

- einmalelastische Binden: 6, 8, $12 \mathrm{~cm}$ für z.B. Druckverbände

- antiadhäsive Wundauflagen für nässende Wunden (z.B. UrgoTül ${ }^{\circledR}$, Allevyn $^{\circledR}$ )

- Schere, Pinzette, Fremdkörperpinzette, Einmalskalpell

- Einmalhandschuhe (steril und unsteril)

- Dextrosetäfelchen (bei Hypoglykämie)

- Tape in unterschiedlichen Breiten (z.B. OSG, Finger)

- Sicherheitsnadeln

\section{Sinnvolle Zusatzausrüstung}

- Merkblatt Erste-Hilfe-Maßnahmen

- Liste des Kofferinhalts

- Dokumentationsbogen für Verletzungen (wichtig nicht nur für evtl. Versicherungsansprüche)
- Set mit kindgerechten Pflastern (ggf. bei Kinder- und Jugendbetreuung)

- Fieberthermometer, Blutzuckermessgerät, Blutdruckmessgerät

- Kugelschreiber

- Taschenlampe

- Papiertaschentücher

- Plastiktüte

- Getränk (z.B. Flasche Mineralwasser)

- Rettungsdecke

- Taschenmesser

- Zahnbox (z.B. Dento-Safe ${ }^{\circledR}$ )

- Sonnenschutzcreme

- Set zur Wundversorgung inkl. Abdeckung (ggf. als steriles Einmalset, SUSI ${ }^{\circledR}$ )

- Wundverschlussstreifen (z.B. SteriStrips $\left.{ }^{\circledR}\right)$, Hautklammergerät

- Gewebekleber (z.B. Histoacryl ${ }^{\circledR}$ )

- Beatmungsmaske/-beutel und GuedelTubus

- Immobilisationssysteme für die Extremitäten in verschiedenen Größen und für verschiedene Gelenke (z.B. SAM Splint ${ }^{\circledR}$, Delta-Cast ${ }^{\circledR}$ )

- Halswirbelsäulenimmobilisationskragen (z.B. Stifneck ${ }^{\circledR}$ )

- unterschiedliche Injektionsnadeln und Spritzengrößen

- Lokalanästhetikum, NaCl-Ampullen

- Entsorgungsbehälter für Injektionsnadeln etc.

- ggf. Minimalset mit Notfallmedikamenten (z.B. für anaphylaktische Reaktion, Epilepsie)

Eventuell ist die Mitnahme der unten aufgeführten Medikamente sinnvoll. Für Betreuung längerfristiger Einsätze (z.B. Trainingslager) sollte eine entsprechende Mengenanpassung erfolgen.

- Medikamente gegen Übelkeit/Erbrechen (z.B. Ondansetron, Dimenhydrinat)

- Medikamente gegen Durchfallerkrankungen (z. B. Imodium ${ }^{\circledR}$ )

- Medikamente zum Ausgleich von Salzund Wasserverlusten (z.B. Elotrans ${ }^{\circledR}$ )

- Medikamente gegen Magenbeschwerden/Sodbrennen (z. B. Magaldrat, Pantoprazol)

- Nasentropfen (z.B. Xylometazolin, $\mathrm{NaCl}$-Lösung/-Spülung)

- Medikamente bei Erkältungskrankheiten (z. B. Halspastillen, Inhalationslösung/-tropfen, Vitamin-C-Brausetabletten)

- Medikamente gegen Asthma (z.B. Salbutamol Dosieraerosol)

- fiebersenkendes Mittel, Schmerzmittel (z.B. Paracetamol, Ibuprofen)

- Medikament gegen Halsschmerzen (z.B. Dolo-Dobendan ${ }^{\circledR}$ )

- Ohrentropfen (z.B. Otalgan ${ }^{\circledR}$ ) 
- Salbe gegen Lippenherpes (z. B. Aciclovir)

- Salbe gegen Pilzerkrankungen (z.B. Clotrimazol)

- Mittel zur Beruhigung (z.B. Baldrian)

Fazit

Der Sportarztkoffer wird i.d.R. individuell zusammengestellt. Von größter Wichtigkeit ist das jeweilige „BetreuungsSetting“. Eine Packliste sollte als eine Art "living document“ angesehen werden.
Kenntnisse der Sportart und sportartspezifischen Anforderungsprofile sind für den Sportarzt unabdingbar.

\section{Literatur}

${ }^{1}$ Im Internet: http://www.din13169.de; Stand: 19.04.2016

2 Im Internet: http://www.din13157.de; Stand: 19.04.2016

${ }^{3}$ Im Internet: www.blsv.de; Stand: 19.04.2016

${ }^{4}$ Im Internet: www.nada-bonn.de; Stand: 19.04.2016
Dr. med. Casper Grim

Leitender Oberarzt

Prof. Dr. Martin Engelhardt

Chefarzt

Klinik für Orthopädie, Unfall- und Handchirurgie

Klinikum Osnabrück

Am Finkenhügel 1

49076 Osnabrück

casper.grim@klinikum-os.de 